\title{
AN EVALUATION OF NUTRITIONAL INDEXES FOR TO PREDICTION THE HOSPITALIZATION DURATION IN ELDERLY CHRONIC KIDNEY DISEASE PATIENTS
}

\author{
B. Buyukaydin ${ }^{1}$, A.T. Isik' ${ }^{2}$ P. Soysal', M. Alay 3 , R. Kazancioglü
}

\begin{abstract}
Objective: Chronic kidney disease and malnutrition are serious and frequently encountered co-morbidities among older patients. We evaluated nutritional status of older pre-dialysis patients and the effect of malnutrition on length of stay (LOS) in hospital. Materials and Methods: 65 years and over 33 hospitalized pre-dialysis patients with glomerular filtration rate between $10-30 \mathrm{ml} / \mathrm{min} / 1.73 \mathrm{~m} 2$ were included. There is no control group. For all patients, biochemical analysis was performed. The malnutrition risk was evaluated with Mini Nutritional Assessment Short-Form (MNA-SF) and Geriatric Nutritional Risk Index (GNRI) and LOS in hospital was recorded. Results: Mean GNRI was $98.4 \pm 12.9$ and the mean MNA-SF was $8 \pm 3.15$. For all patients, mean LOS was $10.58 \pm 9$ days. According to MNA-SF, malnourished patients' LOS was longer and a difference was observed between MNA-SF and GNRI in terms of LOS prediction ( $p=0.005, p=0.230)$. Conclusion: For older pre-dialysis patients, MNA-SF is probably a more sensitive index in terms of LOS in hospital prediction.
\end{abstract}

Key words: Chronic kidney disease, length of stay in hospital, malnutrition, chronic kidney disease, length of stay in hospital, malnutrition, GNRI, MNA-SF.

\section{Introduction}

Length of stay (LOS) in hospital is generally longer in older patients because of many co - morbidities. One of them is malnutrition but despite its high occurence two thirds of the patients remain undiagnosed (1). Another major problem is renal dysfunction and finally chronic kidney disease (CKD). In patients with CKD, many clinical problems lead to prolonged hospitalization but one of these is certainly malnutrition (2). But when encountered a hospitalized older patient, clinicians naturally focus on major systematic problems and malnutrition is usually overlooked (3).

LOS in hospital was associated with plasma volume reduction, accelerated bone loss, sensory deprivation and increased immobilization (4). Prolonged LOS creates malnutrition risk and also malnutrition itself leads to longer hospitalization duration (5). For older hospitalized patients, early detection of malnutrition risk effects the clinical course and also LOS in hospital.

1. Bezmialem Vakif University Faculty of Medicine, Department of Internal Medicine, Istanbul, Turkey; 2. Dokuz Eylul University School of Medicine, Department of Geriatrics, Izmir, Turkey; 3. Bezmialem Vakif University Faculty of Medicine, Department of Nephrology, Istanbul, Turkey

Corresponding Author: Banu Buyukaydin, Bezmialem Vakif University Faculty of Medicine, Department of Internal Medicine, Adnan Menderes Street, 34093 Fatih / Istanbul, Turkey, Fax: +902126217580, Phone: +902124531710,

E-mail: bbuyukaydin@hotmail.com
For detection of this risk we have many nutritional scoring systems. Among them The Mini Nutritional Assessment (MNA) and its short form (MNA-SF) are reliable methods for older patients. These methods' identification power for functionality and LOS in hospital were observed in various studies $(6,7)$.

The Geriatric Nutritional Risk Index (GNRI) is another index and with other variables of nutritional state and it can be used as a tool for malnutrition grading. This index is finally defined a «nutrition related» risk index and has been shown to predict the risk of morbidity and mortality for older patients (7). For hemodialysis patients, lower GNRI index was associated with increased hospitalization duration risk (8).

In hospitalized older patients, using of GNRI in cases where MNA is not applicable, or even using GRNI as a complement to MNA is recommended (9).

However in literature, there are no studies that evaluate GNRI and MNA-SF sensitivity for older predialysis patients. In this study, we evaluated nutrition of pre-dialysis patients with these indexes and investigated possible predictive power of these indexes for LOS in hospital. 


\section{Material and methods}

\section{Study design and participants}

This study was carried out at Bezmialem Vakif University. All new patients admitted to nephrology and geriatrics medical wards with different clinical diagnosis were screened for inclusion. 65 years and over 33 (19 male, 14 female) hospitalized pre-dialysis patients with glomerular filtration rate (GFR) between $10-30 \mathrm{ml} / \mathrm{min} / 1.73 \mathrm{~m}^{2}$ were included. Exclusion criteria were the presence of active neoplasm, hypothyroidism with no treatment, nephrotic syndrome, hepatic failure, malabsorption syndrome, severe heart failure, inflammatory bowel disease, nutrition and swallowing problems and enteral or parenteral nutrition support. Patients under renal replacement were also excluded from the study. Before participation written approval was taken from the patient or the patients' relatives. The study protocol was in accordance with Declaration of Helsinki and was approved by the Institutional Local Ethic Committee (No: 2011/59).

\section{Data collection}

Patients' clinical diagnoses were recorded and patients' weight, height, Body Mass Index (BMI) and biochemical parameters were recorded. Weight was recorded as kilograms using digital electronic scale and BMI were calculated as the weight in kilograms divided by the square of the height in meters and classified according to the World Health Organization criteria. Blood analysis was performed at the central laboratory. Blood urea nitrogen (BUN), creatinine, glucose, serum albumin, total cholesterol, triglyceride, thyroid stimulating hormone (TSH), vitamin $\mathrm{B} 12$, folic acid and $\mathrm{C}$ reactive protein (CRP) levels were tested with Roche Diagnostics $\mathrm{GmbH}$ kits in Roche Hitachi Cobas 8000 device. Patients' GFR was calculated using Chronic Kidney Disease Epidemiology Collaboration (CKD-Epi) and Modification of Diet in Renal Disease (MDRD) formulations (10).

MNA-SF consists of six questions, scored from zero to three and these questions includes declining food intake last three months, weight loss during the last three months, mobility status, psychological stress or acute disease in the past 3 months, neuropsychological problems and BMI. MNA-SF was applied by same researcher and according to MNA-SF, patients are classified as malnourished if their score is between 0 and 7. They are in risk of malnutrition if their score is between 8 and 11 and they are normal if the score is between 12 and 14.

For GNRI, GNRI $=[1.489 \times$ albumin $(\mathrm{g} / \mathrm{L})][41.7 \times(\mathrm{kg} /$ ideal weight)] calculation was used and was recorded for every patient. According to GNRI, patients were classified as at severe risk for malnutrition, if they score are less than 92. They are in low risk if their score is between 92 and 98 and no risk if their score is more than 98.

The duration of hospitalization was recorded for each patient. This data were retrieved from hospital's official information system and recorded in days.

\section{Statistical analysis}

Statistical analysis was performed with using SPSS software version 19.0. Continuous variables defined with statistical characteristics including the mean and standard deviation. In statistical analysis, Spearman test was used for detection of correlation between nutritional indexes. Post-Hoc-Dunn and Kruskal-Wallis analysis were performed for group analysis and correlation coefficient. p less than $<0.05$ was accepted as significant.

\section{Results}

The patients' mean age was $73 \pm 7$ years. Patients' clinical features and nutritional risk analysis between groups are presented in Table 1 . The mean MDRD and CKD-Epi was $18.84 \pm 5.30$ and $17.03 \pm 5.25 \mathrm{ml} / \mathrm{min}$ respectively.

The mean GNRI was detected as $98.4 \pm 12.9$ and the mean MNA-SF was $8 \pm 3.15$. For all patients LOS in hospital was $10.58 \pm 9$ days. Between GNRI and MNA-SF a moderate correlation was detected $(r s=0,402 p=0,018)$. In terms of GNRI, malnourished patients' BMI was lower than other groups $(p=0.000)$. According to GNRI, in patients with low risk group, TSH levels were observed lower than other groups ( $p=0.007$ ).

There was no detected predictive power of these indexes in terms of LOS in hospital. But a difference was found between of indexes in favor of MNA-SF ( $p=0.005$, $\mathrm{p}=0.230$ ).

\section{Discussion}

In this study, we observed elderly hospitalized predialysis patients with co-morbidities and detected a moderate correlation between GNRI and MNA-SF for status of nutrition. However for LOS in hospital prediction, MNA-SF was considered as possible more sensitive nutritional index.

For nutritional screening, MNA-SF is acceptable and reliable index to determine the risk of mortality (11). Also this index can predict functional decline in elderly patients followed in acute care clinics (12). GNRI, another index, was evaluated in large group of patients with different clinical diagnosis. It is a significant predictor of malnutrition and mortality in hemodialysis patients also (13). These indexes were investigated in different patients' groups. One of these studies, with 358 elderly with various diseases, predictive power of these indexes 


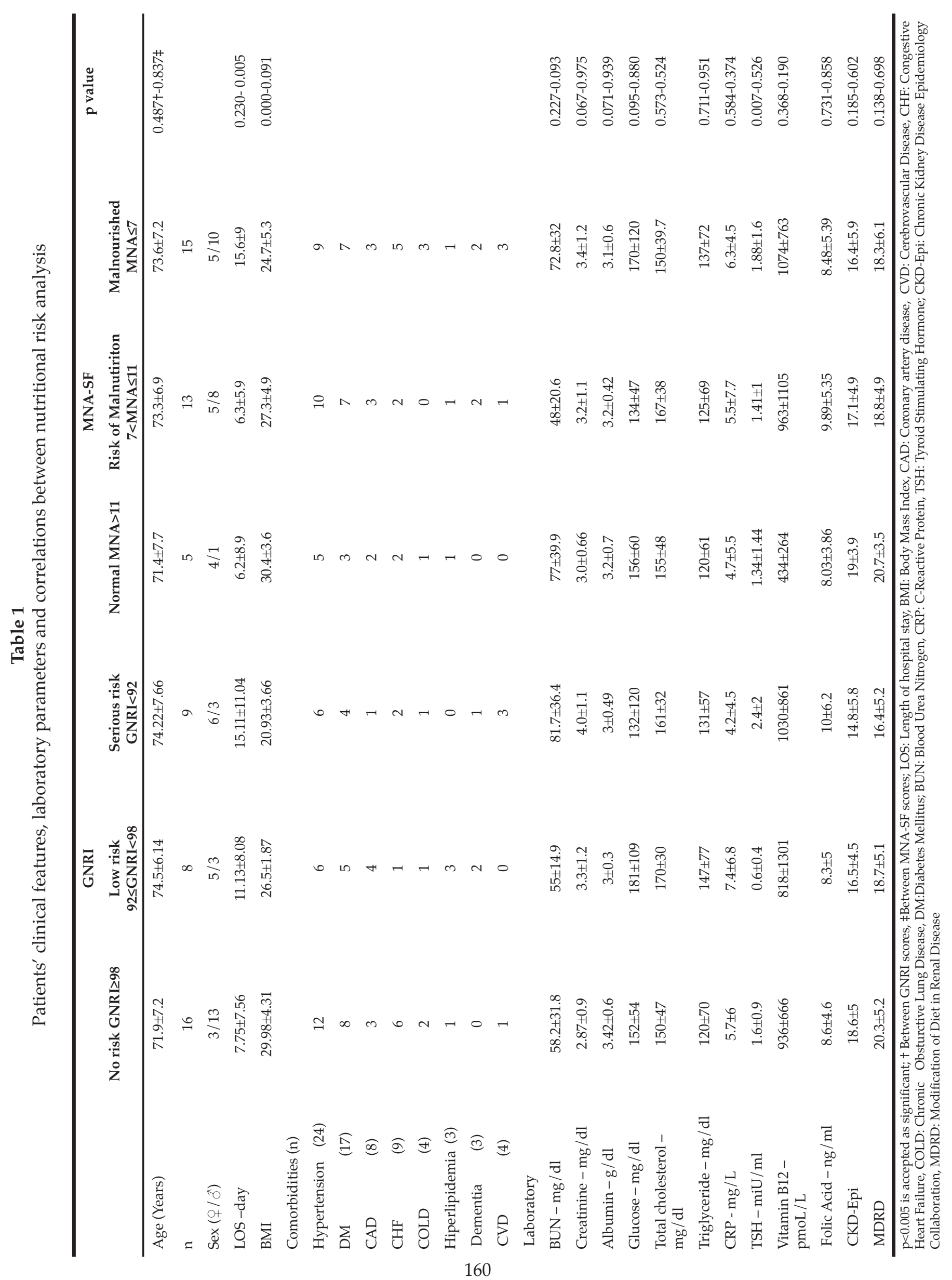


on mortality risk was evaluated. In that study, after 6.5 years follow-up, only nutritional risk evaluated with GNRI was associated with all-cause mortality (14). In another elderly group with heart failure, this index was presented a useful index for functional evaluation and mortality (15). Especially in hospitalized elderly, evaluation together with GNRI and MNA-SF were reported as a proper application (16). But in elderly predialysis patients, the studies that compare nutritional indexes are not sufficient. In this study, we especially evaluated this group of patients and observed a lesser degree correlation between these indexes.

Malnutrition is also associated with prolonged LOS. Thomas et al. found a significant difference between malnourished and at-risk group in terms of LOS. In that study, they used MNA. Low MNA was associated with increased mortality along with LOS in hospital (17). The effect of MNA-SF on LOS was studied in other studies and malnourished and at-risk group, LOS was detected longer and MNA-SF was presented a sensitive index for prediction (18).

Also GNRI was evaluated in terms of this duration and in a few studies the predictive power was demonstrated in different patients' groups. GNRI was determined a significant index in terms of hospitalization probability, inpatient, pharmaceutical and total costs (19). In another larger study, 667 elderly in-patients evaluated, prolonged LOS was detected in patients with high nutritional risk according to GNRI. LOS also was associated with the presence of comorbidities such as cancer and surgical pathologies (20).

LOS is also affected CKD and related problems especially in elderly. Malnutrition is one of these problems. But in literature, especially for this patient group, nutrition indexes' sensitivity for LOS is not known. In this study, we evaluated a specific patient group and observed prolonged LOS in hospital for malnourished patients with a minor statistical difference in favor of MNA-SF.

\section{Conclusion}

The findings of this study indicate that there is a moderate correlation between MNA-SF and GNRI in elderly pre-dialysis patients. But to determine the possible LOS in hospital predictive power of these indexes the large scale and comprehensive studies are required.

Acknowledgements: We would like to thank to all medical assistant team in nephrology and geriatrics clinics, also especially thanks to Omer Uysal M.D and Ali Toprak M.D for biostatistical review.

Conflict of interest statement: There is no conflict of interest.

Ethics Standards: This study was conducted according to the guidelines in the Declaration of Helsinki.

\section{References}

1. Bienia R, Ratcliff S, Barbour GL, Kummer M. Malnutrition in the hospitalized geriatric patient. J Am Geriatr Soc. 1982; 7:433-436.

2. Lacquaniti A, Bolignano D, Campo S, Perrone C, Donato V, Fazio MR, Buemi A, Sturiale A, Buemi M. Malnutrition in the elderly patient on dialysis. Ren Fail. 2009;31: 239-245.

3. Lara-Pulido A, Guevara-Cruz M. Malnutrition and associated factors in elderly hospitalized. Nutr Hosp. 2012;27: 652-655.

4. Creditor MC. Hazards of hospitalization of the elderly. Ann Intern Med 1993;118: 219-223.

5. Finestone HM1, Greene-Finestone LS, Wilson ES, Teasell RW. Prolonged length of stay and reduced functional improvement rate in malnourished stroke rehabilitation patients. Arch Phys Med Rehabil. 1996;77: 340-5.

6. Rubenstein LZ, Harker JO, Salvà A, Guigoz Y, Vellas B. Screening for undernutrition in geriatric practice: developing the short-form mininutritional assessment (MNA-SF). J Gerontol A Biol Sci Med Sci. 2001;56: 366372.

7. Bouillanne O, Morineau G, Dupont C, et all. Geriatric Nutritional Risk Index: a new index for evaluating at-risk elderly medical patients. Am J Clin Nutr. 2005;82:777-783.

8. Baumeister SE, Fischer B, Döring A, Koenig W, Zierer A, John J, Heier M, Meisinger C. The Geriatric Nutritional Risk Index predicts increased healthcare costs and hospitalization in a cohort of community-dwelling older adults: results from the MONICA/KORA Augsburg cohort study, 1994-2005. Nutrition 2011;27: 534-42.

9. Durán Alert P, Milà Villarroel R, Formiga F, Virgili Casas N, Vilarasau Farré C. Assessing risk screening methods of malnutrition in geriatric patients: Mini Nutritional Assessment (MNA) versus Geriatric Nutritional Risk Index (GNRI). Nutr Hosp. 2012;27: 590-8.

10. Froissart M, Rossert J, Jacquot C, Paillard M, Houillier P. Predictive performance of the modification of diet in renal disease and Cockcroft-Gault equations for estimating renal function. J Am Soc Nephrol 2005;16: 763-773.

11. Calvo I, Olivar J, Martínez E, Rico A, Díaz J, Gimena M. MNA® Mini Nutritional Assessment as a nutritional screening tool for hospitalized older adults; rationales and feasibility. Nutr Hosp. 2012;27: 1619-1625.

12. Salvi F, Giorgi R, Grilli A, Morichi V, Espinosa E, Spazzafumo L, Marinozzi ML, Dessì-Fulgheri P. Mini Nutritional Assessment (short form) and functional decline in older patients admitted to an acute medical ward. Aging Clin Exp Res. 2008;20: 322-328.

13. Kobayashi I, Ishimura E, Kato Y, Okuno S, Yamamoto T, Yamakawa T, Mori K, Inaba M, Nishizawa Y. Geriatric Nutritional Risk Index, a simplified nutritional screening index, is a significant predictor of mortality in chronic dialysis patients. Nephrol Dial Transplant. 2010;25: 3361-3365.

14. Cereda E, Pedrolli C, Zagami A, Vanotti A, Piffer S, Opizzi A, Rondanelli M, Caccialanza R. Nutritional screening and mortality in newly institutionalised elderly: a comparison between the geriatric nutritional risk index and the mini nutritional assessment. Clin Nutr. 2011;30: 793-798.

15. Kinugasa Y, Kato M, Sugihara S, Hirai M, Yamada K, Yanagihara K, Yamamoto K. Geriatric nutritional risk index predicts functional dependency and mortality in patients with heart failure with preserved ejection fraction. Circ J. 2008;77: 705-711.

16. Durán Alert P, Milà Villarroel R, Formiga F, Virgili Casas N, Vilarasau Farré C. Assessing risk screening methods of malnutrition in geriatric patients: Mini Nutritional Assessment (MNA) versus Geriatric Nutritional Risk Index (GNRI). Nutr Hosp. 2012;27: 590-598.

17. Guigoz Y. The Mini Nutritional Assessment (MNA) review of the literature-What does it tell us? J Nutr Health Aging 2006;10: 466-485.

18. Charlton KE, Nichols C, Bowden S, Lambert K, Barone L, Mason M, Milosavljevic M. Older rehabilitation patients are at high risk of malnutrition: evidence from a large Australian database. J Nutr Health Aging. 2010;14: 622628.

19. Baumeister SE, Fischer B, Döring A, Koenig W, Zierer A, John J, Heier M, Meisinger C. The Geriatric Nutritional Risk Index predicts increased healthcare costs and hospitalization in a cohort of community-dwelling older adults: results from the MONICA/KORA Augsburg cohort study, 1994-2005. Nutrition. 2011;27: 534-542.

20. Cereda E, Klersy C, Pedrolli C, Cameletti B, Bonardi C, Quarleri L, Cappello S, Bonoldi A, Bonadeo E, Caccialanza R. Clin Nutr. 2015;34: 74-78. 